\title{
Traduire
}

Revue française de la traduction

Traducteurs en blouse blanche

\section{Les pièges interculturels dans la traduction médicale}

\section{Pippa Sandford}

\section{OpenEdition}

1 Journals

\section{Édition électronique}

URL : http://journals.openedition.org/traduire/913

DOI : 10.4000/traduire.913

ISSN : 2272-9992

\section{Éditeur}

Société française des traducteurs

\section{Édition imprimée}

Date de publication : 1 juin 2017

Pagination : 30-39

ISSN : 0395-773X

\section{Référence électronique}

Pippa Sandford, «Les pièges interculturels dans la traduction médicale », Traduire [En ligne],

236 | 2017, mis en ligne le 01 juin 2019, consulté le 30 juin 2019. URL : http://journals.openedition.org/ traduire/913 ; DOI : 10.4000/traduire.913 


\section{Les pièges interculturels dans la traduction médicale}

\section{Pippa Sandford}

Cet article repose sur un atelier que j'ai proposé lors du 11e Séminaire d'anglais médical de la Société française des traducteurs en mars 2016. II présente un aperçu de divers types de problèmes liés aux différences interculturelles tant dans la vie quotidienne que dans les pratiques médicales.

J'ai rencontré la plupart des exemples choisis ici dans ma pratique de traduction et de révision ces dernières années. Les autres, je les ai découverts avec le remarquable Cours d'anglais médical pour traducteurs et interprètes (CMETI) organisé et animé par Karin Band, durant les années 1990. Je lui suis infiniment reconnaissante d'avoir attiré mon attention sur un grand nombre de pièges, bien au-delà de la terminologie. Bien entendu, ce genre d'écueil est présent dans d'autres domaines de spécialisation et dans toutes les paires de langues, aussi est-il essentiel de savoir les repérer afin de les éviter.

Parmi les suggestions proposées pour éviter les erreurs, une bonne maîtrise du sujet l'emporte largement sur la seule recherche terminologique, aussi approfondie soit-elle.

\section{La vie quotidienne}

Une connaissance aussi large que possible de la vie quotidienne dans les pays de vos langues de travail est tout à fait indispensable.

Premier exemple : il est souvent question, dans les textes de pédiatrie, de preschoolers - or, selon l'âge d'entrée à l'école dans le pays concerné, cela peut faire référence aux enfants de moins de quatre ans jusqu'à ceux de sept ans. Le développement physique et mental d'un enfant étant très différent entre quatre et sept ans, il sera judicieux d'ajouter une note précisant l'âge du début de la scolarité dans le pays dont parle l'auteur.

Autre exemple : s'agissant de traduire les réponses à un questionnaire sur les changements de comportement alimentaire chez une personne atteinte de diabète, il peut être utile de savoir 
que lorsqu'un Français dit : " ... maintenant, je m'arrête au fromage ", cela signifie qu'il ne prend plus de dessert ; un Anglais disant qu'il s'arrête au fromage dit tout à fait autre chose, car au Royaume-Uni, le fromage est servi en toute fin de repas.

De même, si à une question liée à son alimentation, un patient anglais répond : / just have my tea in the afternoon, cela peut faire référence à la pause-thé de l'après-midi, tout aussi bien qu'au repas du soir, qu'un autre appellerait dîner ou souper.

Il est donc indispensable de bien connaître les modes de vie dans les pays de vos langues source et de rester à jour très activement dans le cadre de votre formation professionnelle continue. Lisez divers journaux et magazines, suivez des fils Twitter, regardez la télévision et participez à des forums traitant de sujets qui vous intéressent, parallèlement à votre travail.

\section{La pratique médicale}

Les unités de mesure peuvent changer selon les pays - ainsi, pour le temps de prothrombine, l'unité de mesure est la seconde pour les anglophones, tandis qu'en France il peut également être formulé en secondes (" temps de Quick " ou " temps de prothrombine "), mais le " taux de prothrombine " correspond à un pourcentage par rapport à un taux témoin, la norme étant de 70 à $100 \%$. Toutefois, ce pourcentage n'évoquera rien à un lecteur anglais : une valeur normale de $75 \%$ pourrait ainsi lui sembler très basse et donc anormale, ce qui serait une erreur.

En hématologie, l'unité de mesure SI est 109/L, mais nombre d'auteurs français utilisent 'G/L' (G pour giga). Quand les auteurs anglophones utilisent millilitres ( $\mathrm{ml}$ ), les Français utiliseront souvent décilitres $(\mathrm{dl})$ - naturellement, il faut repérer ces différences et, soit modifier les chiffres, soit ajouter une note pour le signaler.

Attention aussi aux mots courants. Regular astigmatism correspond à " astigmatisme régulier ", une forme particulière d'astigmatisme, et en aucun cas à " astigmatisme normal ", qui n'aurait aucun sens!

De même, standard treatment (sans autre précision) peut parfaitement désigner des choses différentes selon le pays, en fonction de la pratique médicale habituelle. Ainsi, un patient atteint de la maladie de Graves (une forme particulière de thyrotoxicose ou hyperthyroïdie) aux ÉtatsUnis recevrait sans doute directement un traitement à l'iode radioactif, tandis que le même patient au Royaume-Uni serait d'abord traité par médication. Ces deux formes de traitement se fondent sur les mêmes données, mais un certain pourcentage de patients ne bénéficiera pas de la possibilité de rémission par carbimazole. Aux États-Unis, le choix est destiné à éviter au patient la déception qu'entraînerait l'échec du traitement au bout de 6 à 18 mois de médication. Au Royaume-Uni, le patient a la possibilité d'essayer d'abord le médicament, qui permet à de nombreux patients d'obtenir une rémission et d'éviter le traitement à l'iode radioactif. 


\section{Les usages différents de termes spécialisés}

Exemple : hyperéosinophilie et eosinophilia.

Les éosinophiles sont un type de cellules sanguines, les globules blancs (appelées aussi leucocytes). S'ils sont présents en trop grande quantité, le terme anglais décrivant cet état est eosinophilia. En cas d'augmentation anormale du nombre d'éosinophiles dans le sang, le terme anglais est hypereosinophilia.

La définition en français est similaire (par ex. "Éosinophilie : Présence de leucocytes polynucléaires éosinophiles supérieure à 500 par mm3; [...] (il serait plus exact de dire : hyperéosinophilie "), mais la plupart des médecins et auteurs français utilisent hyperéosinophilie pour décrire ce qu'un auteur anglais appellerait simplement eosinophilia. Cette confusion est problématique, car si l'éosinophilie est une réaction courante chez des patients hypersensibles ou en cas d'effets secondaires, le terme hypereosinophilia dans un texte en anglais évoque un état beaucoup plus grave. Ainsi, si vous traduisiez I'hyperéosinophilie par hypereosinophilia, l'information transmise serait fausse. Si l'étude de cas précise les valeurs, vous pourrez vérifier le degré de gravité selon les critères des pays concernés et choisir le terme approprié - mais seulement si vous connaissez ce piège.

Cette divergence entre le français et l'anglais dans l'emploi du préfixe " hyper " vaut également pour leucocytose et hyperleucocytose.

\section{Les différences dans l'emploi du langage}

Faux-amis et " amis volages " sont certes l'ordinaire du traducteur, mais il est très facile de les rater quand le délai est serré et que vous ne prenez pas le temps d'une dernière relecture attentive au sens et non limitée à une vérification de la terminologie.

Quelques exemples :

- Promiscuité signifie le fait de vivre nombreux dans un espace réduit, soit overcrowding en anglais, alors que promiscuity concerne la promiscuité sexuelle ; et puisque, de nos jours, promiscuous est perçu comme un jugement négatif, on tend à choisir d'autres formulations, comme " ayant de nombreux partenaires sexuels".

- Intoxiqué - c'est poisoned en anglais, et pas intoxicated (ivre). Ici, la différence est cruciale, surtout dans une situation d'urgence, par exemple un patient inconscient accompagné d'amis francophones qui indiqueraient involontairement, en anglais, qu'il est ivre.

- "L'expertise " désigne un rapport d'expert, expertise, signifie compétence. 
- Le mot " clinique " correspond généralement à private hospital en anglais ; alors que l'anglais clinic peut désigner une consultation ambulatoire à l'hôpital, ou un service spécialisé au sein d'un hôpital (eye clinic / service ophtalmologie, etc.).

- "Perfusion " se traduit le plus souvent par infusion, sauf dans certains cas précis.

- "Infiltrée " peut se traduire de plusieurs façons - à propos des artères, la phrase " 3 axes de jambe infiltrés mais perméables " signifie que les vaisseaux montrent des signes d'athérosclérose ou plaque, avec divers niveaux de sténose ; infiltration serait employé au stade de l'examen microscopique plutôt que clinique. Le contexte est essentiel ; c'est une évidence, mais qui impose une vigilance accrue quand les délais sont serrés.

- On pourrait écrire un article entier sur le mot " prothèse ", qui se traduit ponctuellement par prosthesis en anglais, mais qui, le plus souvent, serait rendu par d'autres termes complètement différents et sans doute plus précis.

- "Mal au foie " correspond le plus souvent à stomach ache ou quelque chose d'approchant, mais je l'ai rencontré comme traduction, fautive, de liver disorder, une atteinte beaucoup plus grave du foie. La connaissance de la maladie en question aurait évité ce contresens.

- Délai $\neq$ delay - " un délai de deux semaines ", ne veut pas du tout dire : there is a two weeks' delay (= deux semaines de retard).

Mieux vaut les repérer et en faire un répertoire à consulter régulièrement pour rester à jour.

\section{Les nouvelles technologies}

À nouvelles technologies, nouvelle terminologie :

Les termes adoptés par les différents pays sont dissemblables, peut-être parce que focalisés sur des aspects techniques différents et ils peuvent en outre changer rapidement à mesure que la technologie devient familière.

Exemple : les anticoagulants oraux, des médicaments prescrits pour empêcher la formation de caillots sanguins afin d'éviter une attaque ou un arrêt cardiaque. Les patients sous anticoagulants étant nombreux, le sujet revient souvent dans les rapports médicaux, les discussions sur le choix du meilleur médicament, les études de nouveaux médicaments et les rapports d'essais cliniques.

Encore récemment, les principaux médicaments de cette famille présentaient nombre d'inconvénients, notamment la contrainte d'un suivi régulier par des analyses de sang pour vérifier l'adéquation du dosage. Mais plusieurs nouveaux anticoagulants ont été mis sur le marché au cours des dernières années (par ex. dabigatran, apixaban, et rivaroxaban), tous annoncés 
plus simples et plus sûrs que les précédents (réduction des risques de saignements), et n'impliquant pas de surveillance biologique récurrente.

On les a appelés new oral anticoagulants, novel oral anticoagulants (NOACS) ou parfois direct oral anticoagulants (DOACS) (du fait de leur action directe contre les facteurs de coagulation, à la différence de certains médicaments antérieurs).

En France on les a d'abord appelés " nouveaux anticoagulants oraux " (NACO), avant de préférer " anticoagulants oraux directs " ou AOD, plus précis, car ils ne seront pas toujours nouveaux et ont bien une action directe sur les facteurs de coagulation. Cependant, le volume d'informations provenant des États-Unis, du Royaume-Uni et d'autres pays anglophones a été tel que l'appellation anglaise, NOACs, deviendra probablement la plus courante. La terminologie n'est pas encore fixée. Des directives françaises récentes ont, en effet, clairement recommandé la forme NACO au lieu d'AOD, pour s'aligner sur l'usage anglais.

Situation classique avec les nouveautés techniques : les termes changent très vite à mesure que la nouveauté se banalise. Cela s'est produit en anglais avec l'apparition des angiotensinconverting enzyme inhibitors (ACE inhibitors); la formulation d'origine était converting enzyme inhibitors (CEls), en français : inhibiteurs de l'enzyme de conversion (IEC). Vos clients peuvent avoir une nette préférence pour l'un de ces termes (qui peut très bien, avec le temps, devenir une préférence tout aussi nette pour l'autre terme), et si vous faites le mauvais choix, votre traduction pourra être jugée datée, voire peu compétente.

En outre, l'apparition de ces nouveaux médicaments a influencé la façon dont on parle des anticoagulants oraux en général, puisque les possibilités ne se limitent plus au choix entre inhibiteurs plaquettaires (aspirine et clopidogrel) et antivitamines K (warfarine). Le terme " anticoagulant oral " était autrefois employé indifféremment à propos de tout traitement destiné à empêcher les caillots, mais on tend désormais à choisir des désignations plus précises comme " inhibiteurs plaquettaires ", " antivitamines K " (AVK) et " nouveaux anticoagulants oraux " (NOAC). On peut facilement confondre ces catégories si l'on ne comprend pas ce qui les différencie ni les effets qu'ont ces médicaments, et il est bien sûr crucial de savoir les distinguer lorsqu'il s'agit de comparaison au cours d'essais cliniques requis par l'autorité de réglementation pour vérifier leur innocuité, par exemple.

Les enjeux (cliniques et financiers) sont souvent très importants lorsqu'apparaît une avancée technique, avec de gros besoins de traduction quand fabricants et utilisateurs s'emploient à identifier les groupes de patients auxquels le produit sera le plus bénéfique et ceux (le cas échéant) qu'il exposerait à des effets secondaires. Dans le cas des anticoagulants oraux, le bénéfice était manifeste mais les effets secondaires et les conséquences d'un surdosage pouvaient être très graves, ce qui a généré nombre d'études, de rapports d'évaluation, d'articles, etc. à traduire. 
Autre conséquence des rapides évolutions linguistiques autour des nouvelles technologies: l'obsolescence d'une traduction ou d'une entrée de glossaire, qui peut vous échapper si vous ne travaillez pas régulièrement dans le domaine. Vous risquez alors d'employer un terme approuvé par l'agence ou le client quelques mois plus tôt, mais qui aura été remplacé depuis. Rester à jour est essentiel, et le simple fait de dater vos entrées de glossaire, par exemple, peut y participer.

\section{Nouvelles technologies $\mathbf{2}$ - les dérivateurs de flux}

L'anévrisme intracrânien (ou anévrisme cérébral) est la dilatation localisée d'un vaisseau sanguin du cerveau qui rend la paroi plus fine et plus fragile et provoque une déformation, comparable à une petite poche, comme sur le schéma ci-dessous :

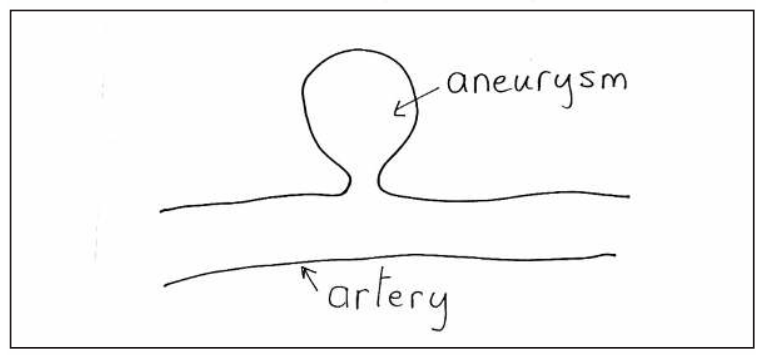

Figure 1 - Artère avec anévrisme.

L'anévrisme peut se rompre ou se fendre et le sang va se répandre dans les tissus alentour, ou bien provoquer une pression sur un nerf ou une partie du cerveau.

Un traitement non invasif consiste à introduire un cathéter jusqu'à l'anévrisme, puis, à l'aide d'un micro cathéter, insérer dans ce cathéter des spires de fil métallique qui viendront remplir le sac anévrismal. C'est ce que l'on appelle l'embolisation par voie endovasculaire.

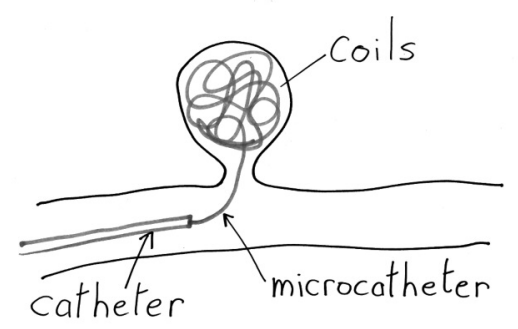

Figure 2 - Anévrisme après embolisation endovasculaire. 
Le procédé consiste à provoquer l'embolisation, c'est-à-dire à faciliter la formation d'un caillot de sang dans l'anévrisme afin d'exclure ce dernier de la circulation sanguine dans le cerveau. On peut également choisir d'insérer un stent (mot anglais employé tel quel dans le langage médical français) dans le vaisseau parent, toujours pour détourner le flux sanguin de l'anévrisme et le maintenir dans l'artère, comme ceci :

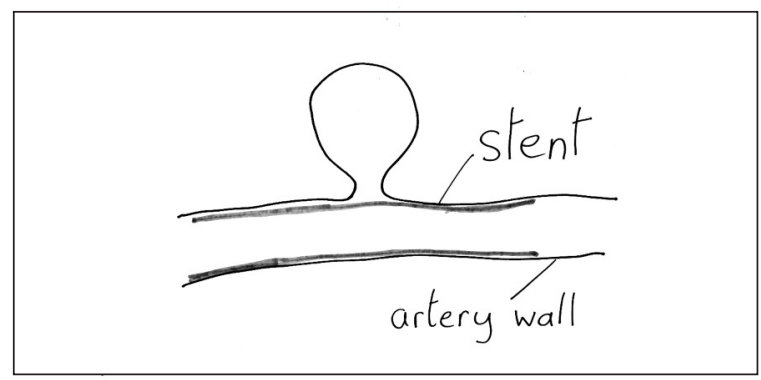

Figure 3 - Anévrisme avec stent pour en détourner le flux sanguin.

Ce type de stent est souvent appelé flow diverter en anglais, et " stent intracrânien " en français (on utilise aussi parfois intracranial stent en anglais).

Une entreprise a amélioré la méthode classique d'embolisation endovasculaire avec un dispositif qui vient perturber le flux sanguin dans l'anévrisme même. Voici la description fournie par l'entreprise, en anglais et en français, de ce dispositif :

"Building upon the foundation of endovascular embolic coils, the WEB device is an intrasaccular flow disruptor that is implanted into a ruptured or unruptured intracranial aneurysm to promote rapid, peri-procedural stasis."

"Fondé sur des spires d'embolisation par voie endovasculaire, le dispositif WEB est un dérivateur de flux intrasacculaire, qui est implanté dans un anévrisme intracrânien rompu ou non rompu, pour favoriser une stase péri-procédurale rapide. "

Le flow disruptor anglais devient "dérivateur de flux " en français - littéralement flow diverter, qui correspond à "stent intracrânien " en français.

Ces deux dernières années, j'ai rencontré d'autres traductions françaises, comme " perturbateur de flux " et " dispositif d'embolisation " pour flow disruptor, mais elles ne semblent pas s'être imposées. 


\section{Autre terminologie nouvelle}

II y a aussi la terminologie régie par les directives de l'Union européenne (UE). Des termes officiels peuvent être modifiés ou se voir attribuer une autre signification et, si vous l'ignorez, votre traduction sera littérale au lieu de respecter la nouvelle définition officielle. Par exemple, temporary halt (" arrêt temporaire " en français) semble assez clair, mais a désormais un sens particulier et renvoie à la suspension d'un essai clinique déclenché par le promoteur, la " suspension " étant, elle, du fait d'un État membre.

J'ai trouvé ces informations en consultant l'excellent blog d'Emma Goldsmith Signs and Symptoms of Translation (https://signsandsymptomsoftranslation.com/) à propos d'une analyse de changements dans la terminologie résultant d'une nouvelle réglementation de l'UE sur les essais cliniques. Une lecture régulière de ce genre de blog spécialisé, dans le cadre de votre autoformation continue, peut vous aider à rester à jour ; pour la langue anglaise, vous pouvez suivre Grant Hamilton (Anglocom - @anglais) et François Lavallée (F. Lavallée (Mag @Magistrad Plus) sur Twitter - deux traducteurs chevronnés dont les fils Twitter regorgent de conseils. II y en a bien d'autres.

\section{On dirait...}

On décrit les choses différemment, selon sa culture. Sachant que les radiologues ont l'habitude de baptiser les images sur les clichés en fonction de ce que leur évoquent leurs formes, ces métaphores peuvent poser quelques sérieuses " colles " au traducteur.

Voici quelques usages courants en anglais, selon un site de radiologie : pour décrire une opacité pulmonaire " en ailes de papillon ": bat-wing appearance (litt., en ailes de chauve-souris) ; l'aspect de la colonne vertébrale dans la spondylarthrite ankylosante : bamboo spine (soit colonne de bambou ou en rail de chemin de fer) ; maiden waist sign (en taille de jeune fille) pour la déformation décrite en français comme une attraction vers la ligne médiane de l'uretère au niveau de la sténose ; et Mickey Mouse ears (en oreilles de Mickey).

Le problème est que ce qui semble évident et significatif pour un radiologue anglophone n'aura souvent aucun sens pour son confrère français, et inversement.

Il vous faut donc savoir à quoi correspondent les descriptions en question afin de pouvoir les décrire en termes courants, compris du lecteur. Pour la radiologie, il y a d'excellents sites web comme Learning Radiology (http://www.learningradiology.com/radsigns/radsignstoc.htm) et Radiopaedia (https://radiopaedia.org).

Parfois le terme peut servir pour décrire deux choses complètement différentes. Ainsi, en radiologie, les " oreilles de Mickey " peuvent décrire l'écartement des ailes iliaques du pelvis chez une personne atteinte de trisomie 21 ou, sur un scanner, l'hyperfixation sur les pédicules 
et l'apophyse épineuse du marqueur radioactif, dans la maladie osseuse de Paget. Elles peuvent aussi décrire l'hydrocéphalie, plutôt qu'une atrophie de la substance blanche du cerveau, et à l'échographie, évoquer une anencéphalie, ou bien confirmer la normalité de l'anatomie de l'espace porte. II faut donc toujours se concentrer sur ce que décrit effectivement l'auteur, sans se contenter de reprendre ce qui semble plausible dans vos mémoires de traduction ou glossaires, ni céder à la tentation d'utiliser une équivalence trouvée sur internet. J'ai ainsi rencontré la référence aux " oreilles de Mickey " traduite par les ailes iliaques de la trisomie 21, alors que le reste de l'étude concernait manifestement un patient atteint de la maladie de Paget.

Et puis, bien sûr, dans un échange écrit entre médecins, l'ellipse est de mise : Mickey Mouse present (Mickey est là) commente une radiographie, pas la présence de Mickey ; double-bubble (double bulle) à propos d'un nouveau-né résume le double-bubble sign (soit la dilatation de l'estomac et du duodénum proximal) présent sur une radiographie ou une échographie ; et dromedary hump ++ (bosses de dromadaire) à propos d'un patient souffrant de problèmes rénaux signifie que la radiographie montre d'importants renflements, souvent baptisés « bosses de dromadaire ", sur le bord latéral du rein gauche.

Dans un tout autre registre, Mickey Mouse peut qualifier quelque chose de bâclé - a Mickey Mouse repair, c'est une réparation (mal) bricolée.

La dermatologie est un autre domaine où l'on rencontre des descriptions diverses d'un même aspect, et les lésions seront donc évoquées par des images très différentes selon les langues.

Ainsi, la description des lésions de l'érythème polymorphe (erythema multiforme) évoquera sans doute l'aspect caractéristique de cercles concentriques. En anglais cela donnera target lesions (ou bullseye ou iris lesions) et en français "lésions cocardes ", et au vu de l'absence de cockade lesions dans les textes anglais, vous comprendrez que l'auteur français qui les décrit en anglais par so-called cockade lesions ne s'est pas trop foulé (un travail de Mickey). Le terme " en cocarde " apparaît aussi dans la description d'échographies et cet " aspect en cocarde " (ou aspect en cocarde avec centre hyperéchogène) est simplement traduit par hypoechoic rim.

\section{Les éponymes}

Nombre de maladies, pathologies et manœurres ont été nommées d'après la personne qui les a découvertes, ce qui peut engendrer une certaine confusion, différents pays utilisant des noms différents. Ainsi, les médecins allemand Karl Adolph von Basedow et irlandais Robert Graves ont décrit séparément l'association de l'exophtalmie (yeux saillants) avec le goître (hypertrophie de la thyroïde) qui se produit chez les personnes atteintes d'une forme précise de thyrotoxicose (ou hyperthyroïdie). La maladie est donc appelée maladie de Basedow en Europe continentale, 
et maladie de Graves aux États-Unis et au Royaume-Uni, mais il existe nombre d'autres versions selon les langues (par ex. maladie de Parry, de Begbie, de Flajani, de Marsh et syndrome de Flajani-Basedow), selon les différents médecins ayant aussi remarqué l'association.

Parfois, les noms sont similaires dans les deux langues, mais pas identiques :

Colles fracture (EN) et fracture de Pouteau-Colles (FR) ; Henoch-Schönlein purpura (EN) et Purpura (ou maladie) de Schönlein-Henoch (FR) ; Chiari's disease (EN) et maladie de BuddChiari ou thrombose des veines hépatiques (FR).

On peut aussi avoir un éponyme dans une langue et pas dans l'autre : Abrami's disease (EN) correspond à " anémie hémolytique acquise " en français.

Parfois, il y a des surprises : la lésion congénitale asymptomatique de communication interventriculaire ou " maladie de Roger " (FR) reste " maladie de Roger " Roger's disease en anglais.

La plupart des revues demandent précisément que les appellations éponymes soient explicitées ou que l'on donne aussi un terme plus courant ou universel.

\section{Conclusion}

Comment éviter ces divers pièges ? Deux suggestions :

1) Maintenir activement à jour votre connaissance du sujet, par la formation continue.

2) Rester vigilant pour détecter les problèmes, par exemple en prenant le temps de lire la traduction entière comme un texte qui vous serait destiné, sans vous contenter de vérifier les mots. C'est une autre forme de révision.

Avec une lecture critique, vous saurez comment bien cibler vos recherches - un gage d'efficacité. Cette méthode aurait sans doute évité à un traducteur de choisir " maladie de Charcot " (sclérose latérale amyotrophique) pour Charcot + , alors que l'étude traitait manifestement de l'arthropathie diabétique (ou neurogène, ou de Charcot). Le client n'a guère apprécié.

Traduit de l'anglais par la rédaction

Pippa Sandford a exercé à plein temps la traduction médicale (français et italien vers l'anglais) en indépendante pendant plus de trente ans. Elle a été responsable de la rédaction du bulletin du Medical and Pharmaceutical Network de l'ITI pendant plusieurs années, et elle a enseigné la traduction (en troisième cycle d'études universitaires) à Salford, Angleterre. 